\title{
Cenplesto
}

\section{O processo de atenção e o letramento informacional}

\author{
Kelley Cristine Gonçalves Dias Gasque \\ Doutora; Universidade de Brasília, Brasília, DF, Brasil; \\ kelleycristinegasque@hotmail.com
}

\begin{abstract}
Resumo: O presente artigo constitui-se em uma revisão de literatura, de natureza exploratória e seletiva, com objetivo de mapear estudos que envolvem o letramento informacional e o processo de atenção, levantar possíveis tópicos de pesquisas e identificar formas de regulação da atenção. Parte-se do pressuposto que a atenção é um processo fundamental na aprendizagem dos conteúdos de letramento informacional, bem como na realização de atividades e das tarefas de pesquisa. Os resultados mostram que manter a atenção é fundamental para as tarefas cognitivas humanas, e consequentemente, o letramento informacional. As pesquisas na área de ciência da informação sobre esse tópico ainda são incipientes e precisam de investimentos. De maneira geral, na literatura, reconhece-se a possibilidade de melhorar o foco por meio da regulação cognitiva ou comportamental.
\end{abstract}

Palavras-chave: Letramento informacional. Atenção. Autorregulação. Aprendizagem.

\section{Introdução}

No mundo contemporâneo, em que há excesso de informações, torna-se impossível para o ser humano processar todas as informações do ambiente, por serem desnecessárias e também pouco econômico. Além disso, Goleman (2014) afirma que, atualmente, há o empobrecimento do processo de atenção decorrente da quantidade de informação que precisa de compreensão. Na década de 70, Herbert Simon (1971), que recebeu o prêmio nobel da economia, previu que o mundo se tornaria cada dia mais rico em informações, o que consumiria demasiadamente a atenção dos indivíduos. Nesse sentido, o autor afirma que a baixa atenção é consequência da riqueza das informações. 


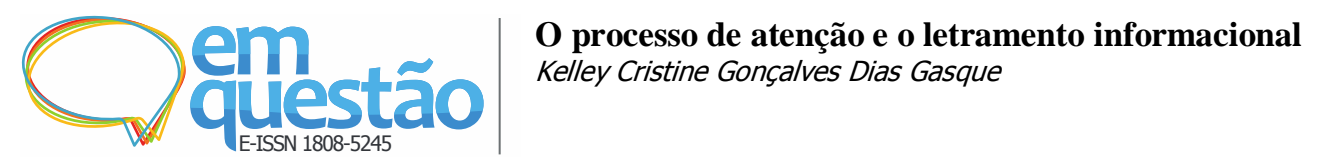

A aprendizagem do letramento informacional (LI) possibilita desenvolver competências necessárias para saber buscar e usar a informação para algum propósito. A aprendizagem envolve a aquisição de conteúdos, que abrange:

a) o desenvolvimento das capacidades de identificação de problemas, delimitação das informações necessárias;

b) acesso e avaliação da informação;

c) uso da informação de forma efetiva para atingir objetivos específicos;

d) comunicação da informação e compreensão dos aspectos econômico, legal e social da informação.

Para que os estudantes se apropriem do conteúdo e desenvolvam as capacidades informacionais, é preciso considerar vários aspectos da aprendizagem, em especial, o processo de atenção. A atenção, grosso modo, pode ser compreendida como o processo de estar presente de corpo e mente, consciente do que se vivencia em determinada situação. Em geral, os estudos sobre o assunto mostram que, muitas vezes, indivíduos estão presentes de corpo, mas com a mente divagando por outros mundos e espaços. Contudo, de acordo com Goleman (2014), estar atento é a chave para o sucesso. Isto é, a atenção determina o que se vê e o que se aprende. Atualmente, observa-se que a quantidade de informação recebida pelos indivíduos os tornam desorganizados e a atenção torna-se menos eficiente.

A atenção refere-se à capacidade do sujeito de controlar e regular a manutenção do foco, em determinado objeto, na realização das tarefas cognitivas, por exemplo, aquelas relacionadas ao LI. Assim, a atenção é condição sine qua non para a aprendizagem e para o desempenho das tarefas de informação. Nesta perspectiva, não se considera o processo de atenção do ponto de vista patológico, mas a partir da visão de pessoas sem diagnóstico médico de défict de atenção. As queixas patológicas de falta de atenção podem ter causas múltiplas como as questões relacionadas ao desenvolvimento, à estrutura orgânica ou ao emocional e relacional (DE-NARDIM; SORDI, 2007). 


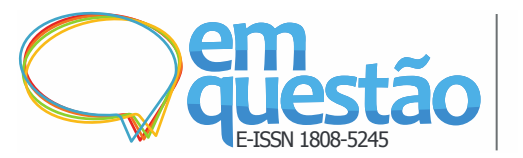

Portanto, ao reconhecer a importância da atenção para o processo de aprendizagem, e em especial, para o LI, realizou-se revisão de literatura, do tipo narrativa, com o objetivo de: (1) levantar pesquisas que relacionem o processo de atenção ao letramento informacional; (2) identificar formas de regulação da atenção; e (3) sugerir tópicos de pesquisas sobre o assunto.

\section{$2 \mathrm{O}$ processo atencional}

Por meio da atenção, o ser humano pode focalizar determinados aspectos do ambiente e ignorar o que for dispensável. Isto é, a atenção é o processo de estar com o foco em um estímulo específico. Sobre isso, Willian James argumenta que a atenção é o oposto da distração.

Todos sabem o que é a atenção. É a posse pela mente, de forma clara e viva, de um entre vários objetos ou sequências de pensamento que parecem simultaneamente possíveis. A essência da atenção é constituída pela focalização, concentração e consciência. (JAMES, 1890, doc. não paginado).

Sternberg (2000) explica que a atenção diz respeito ao fenômeno de processar ativamente uma quantidade limitada de informação dentre as informações disponíveis, o que possibilita economia dos recursos mentais. Dessa forma, a atenção é o processo responsável por fazer a triagem dos vários estímulos visuais, auditivos, olfativos ou táteis recebidos, selecionando-os, de acordo com os objetivos e vivências do indivíduo.

A atenção permite monitorar as interações entre homem e o ambiente; vincular as memórias ao presente para dar continuidade às experiências; controlar e planejar as ações. O estudo da atenção envolve questões sobre consciência, processos controlados e automáticos, habituação, funções da atenção e teorias da atenção. A consciência, apesar da dificuldade de conceituála, pode ser entendida, grosso modo, como a capacidade de monitorar, avaliar e filtrar as informações ambientais através da mente (GASQUE, 2017).

As funções principais da atenção, de acordo com Sternberg (2000) são: 


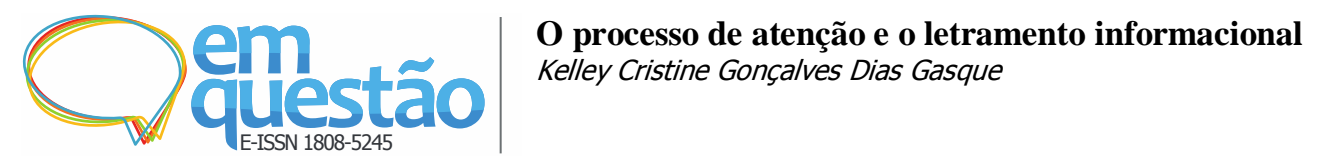

a) atenção seletiva/sustentada - capacidade de manter a atenção em determinado estímulo ao ignorar outros estímulos ou diminuir a ênfase sobre eles;

b) vigilância - capacidade de esperar atentamente para detectar o aparecimento de um estímulo específico;

c) sondagem - capacidade de procurar estímulos específicos;

d) atenção dividida - capacidade de distribuir os recursos atencionais disponíveis para melhorar o desempenho em mais de uma tarefa ao mesmo tempo.

William James (1890) diferenciou o modo de atenção ativa - controlada de cima para baixo (top-down) pelos objetivos e expectativas pessoais, da atenção passiva - controlada de baixo para cima (bottom-up) por estímulos externos, por exemplo, um som alto. Eysenck e Keane (2007) explicam que os modos de atenção podem ser divididos entre a atenção seletiva (focalizada) e a dividida. No primeiro caso, a atenção é seletiva, quando processa apenas um estímulo, que pode ser auditivo ou visual. Por sua vez, a atenção dividida processa vários estímulos, considerando a similaridade e dificuldade da tarefa, bem como o grau de automação (prática) da tarefa. Para este tipo de pesquisa, apresentam-se aos indivíduos dois ou mais estímulos, solicitando-lhes que respondam a todos. Este estudo propicia a compreensão sobre as limitações da atenção.

Várias teorias foram propostas para explicar as funções da atenção ao longo do tempo. Muitos pesquisadores tentam compreender como as pessoas filtram as informações irrelevantes e decidem focar em uma nova informação. Isso pode ser vivenciado, por exemplo, quando se está em uma sala cheia de pessoas e de conversas, e é possível prestar atenção na pessoa com quem se conversa, mas ao mesmo tempo ouvem-se pedaços de conversas das outras pessoas que estão por perto. Uma maneira de estudar a atenção seletiva é por meio da tarefa de sombreamento. Nessa tarefa, o indivíduo usa fones de ouvido, mas ouve informações diferentes reproduzidas em cada orelha. $\mathrm{O}$ indivíduo deve 


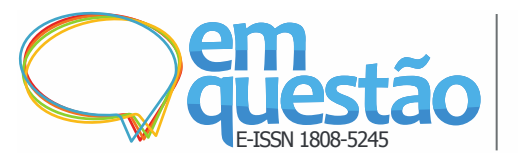

repetir tudo o que é dito em um ouvido, isto é, prestar atenção no som que sai para um ouvido e ignorar o som reproduzido no outro.

Há três teorias principais, do ponto de vista histórico, que tentam explicar o processo de atenção. A primeira é a teoria seletiva de Broadbent, de 1958. Para esse pesquisador, as informações do ambiente passam pelo registro sensório das pessoas, onde são registradas ou armazenadas. Isso inclui qualquer tipo de informação como palavras, cliques, sirenes e vários outros tipos de entradas. Depois, a informação é transferida diretamente para o filtro seletivo, que identifica o que deve ser atendido de acordo com as características físicas básicas. Por exemplo, o filtro seletivo pode identificar a voz, o tom, a velocidade e o sotaque, características que as pessoas não precisam entender para identificar a informação. Em seguida, a informação selecionada segue para o processo de percepção, que propicia significado à informação. Assim, enquanto o filtro seletivo identifica o tom da pessoa em quem se quer prestar atenção, o processo perceptual identifica isso como a voz de algum conhecido e dá significado para as palavras. Contudo, a teoria de Broadbent (1958) apresenta alguns problemas, pois se alguém filtrar completamente a informação não focalizada do meio antes de dar significado, não seria possível a pessoa identificar o próprio nome. Mas, quando isso ocorre, a pessoa ouve imediatamente o nome dela, até do outro lado da sala, mesmo sem estar atento àquela conversa antes. Este fenômeno é conhecido como a "festa do coquetel" e trouxe novos insights aos pesquisadores (EYSENCK; KEANE, 2007; STERNBERG, 2000).

Em 1963, Deutsch e Deutsch, propuseram uma teoria da denominada seleção tardia, que moveu o filtro seletivo, proposto por Broadbent, para depois do processo perceptivo. Ou seja, a teoria dos autores parte do princípio de que as pessoas registram o significado de todas as informações do ambiente, mas o filtro seletivo decide o que é relevante para ser focalizado. Porém, considerando que os recursos atencionais são limitados, questiona-se a necessidade de gastar tanta energia para dar significado para coisas que não serão processadas. Treisman, em 1964, propôs a teoria da atenuação sobre a atenção seletiva, em que a ideia seria trocar o filtro completo para um atenuador, que reduz os estímulos. O autor enfatiza que a função do filtro atenuador é a redução e não a 


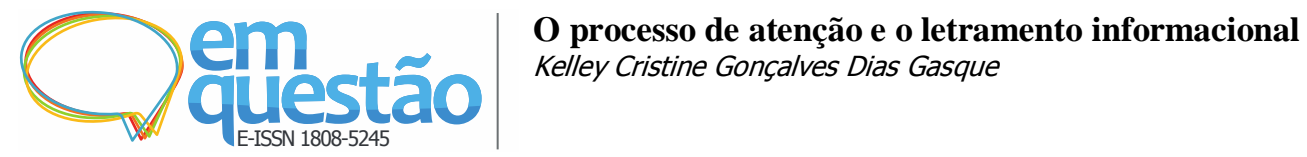

eliminação completa da entrada de informações no ouvido, em que algo disso chega ao processo perceptual. As pessoas dão significado para as informações ambientais, sem, porém, dar prioridade à elas. Se houver a percepção de que as informações são importantes, a pessoa troca a atenção e enfraquece o que ouvia anteriormente. A dificuldade da tarefa pode ser afetada pelo momento em que a filtragem ocorre e pelo tempo de duração (EYSENCK, KEANE, 2007; STERNBERG, 2000).

As teorias ainda suscitam debates sobre qual delas explica melhor a atenção seletiva, visto que a atenção é crucial para as demais funções cognitivas. Se as crianças não conseguissem atender vozes humanas e filtrar pássaros piando ou cachorros latindo, seria quase impossível aprenderem a falar. E se não houvesse como centrar-se, de novo, em informações não atendidas, quando um carro viesse na direção de alguém ou se alguém gritasse "fogo!" não seria possível compreender a informação (STERNBERG, 2000).

Ainda de acordo com o autor supracitado, as teorias mais recentes se encaminham para a ideia de partilha de recursos limitados da atenção e tentam explicar como várias tarefas são realizadas ao mesmo tempo. Isto é, as pessoas têm um recurso limitado de atenção, que é distribuído em consonância com as exigências observadas nas tarefas. Esta teoria foi bastante criticada por ser muito ampla e vaga. No entanto, o autor acredita que ela pode ser uma complementação das teorias dos filtros para explicar alguns aspectos da atenção. Sobre a questão da atenção dividida, os experimentos mostram que as pessoas dividem melhor a atenção quando as tarefas competidoras são de diferentes modalidades.

Em relação aos métodos para avaliação da atenção, Lima (2005) elenca os principais, quais sejam: implantação de microeletrodos em macacos para verificar os efeitos neurais, Pósitron Emission Tomography - PET, ressonância magnética e técnicas de Neuroimagem, bem como os testes neuropsicológicos e clínicos. No entanto, tradicionalmente os testes mais são usados para medir o fenômeno da atenção são Stroop Color Word Test ${ }^{1}$, Trail Making Test ${ }^{2}$, testes de Cancelamento (TC) ${ }^{3}$, Tower of London ${ }^{4}$, dentre outros. Porém, destaca-se que 


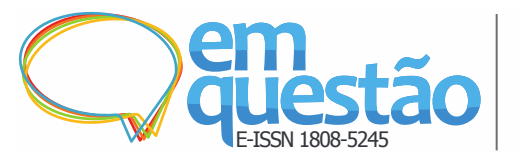

a literatura é escassa de referência em relação ao desempenho dos instrumentos (LIMA, TRAVAINI, CIASCA, 2009).

De-Nardim e Sordi (2007) argumentam que a atenção, na atualidade, pode remeter tantos aos caminhos biológicos e fisiológicos, quanto aos psicológicos e culturais. Para as autoras, há um paradoxo no que diz respeito ao conceito de atenção requerida para a aprendizagem como função cerebral mensurável e alguns estudos mais recentes que apontam a atenção como função de caráter histórico.

Goleman (2014) argumenta que a ciência da atenção transcende a ideia de vigilância. Para o autor, a competência com que um sujeito realiza determinada tarefa vincula-se à capacidade de atenção, e ainda assim, ela é um "recurso subestimado e pouco percebido" (GOLEMAN, 2014, p. 11). Ele conclui que a vida plena depende do foco interno, foco no outro e o foco externo. O primeiro relaciona-se com as intuições, valores e decisões pessoais. $\mathrm{O}$ segundo diz respeito à interação com as pessoas e o terceiro diz respeito ao mundo.

Portanto, a atenção é um dos alicerces para a compreensão, adaptação e transformação do mundo. Muitas vezes, o cérebro está sintonizado com o ambiente, mesmo que não se tenha consciência disso. O que se sabe é que para adquirir o conhecimento consciente de alguma coisa, é necessário prestar atenção. A intensidade da atenção relaciona-se ao nível de interesse, atenção e ansiedade do indivíduo (RATEY, 2002, p. 128).

\subsection{Regulação da atenção}

Uma pessoa, ao reconhecer a necessidade de informação para realizar um projeto, pode iniciar o processo de busca da informação na internet, em função da facilidade de acesso. A internet pode se constituir em um grande distrator da atenção, por meio dos e-mails, anúncios e milhares de hiperlinks. Se o leitor não consegue manter o foco, o processo pode sofrer uma disrupção. Dependendo da especificidade do assunto, a busca de informação pode demorar várias horas ou 


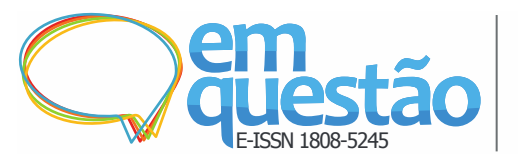

dias de pesquisa, constituindo-se uma tarefa complexa, que depende do uso de estratégias como identificação de palavras-chave e descritores; uso de operadores booleanos, truncagem; navegação por vários bancos de dados, em geral, com estruturas diferentes, além de avaliações e seleção das informações. De posse das informações, é necessário transformá-las em conhecimento. Isso ocorre por meio do planejamento e aplicação de estratégias de estudo e leitura; representação da informação por meio de resumos, esquemas e mapas conceituais. Ao fim do processo, é importante o planejamento da comunicação da informação, o que requer organizar as tarefas, considerando os objetivos da pesquisa, os meios de divulgação, o conhecimento das normas para estruturar a informação, etc.

O processo de pesquisa, descrito acima, requer do indivíduo competências para lidar com a informação, além da capacidade de atenção seletiva para um desempenho eficaz. Os fatores que influenciam a atenção estão, de acordo com revisão realizada por Lima (2005), relacionados ao contexto em que o indivíduo vive, às características dos estímulos, à motivação, à expectativa, à relevância da tarefa, ao estado mental e às experiências anteriores. Nesse sentido, Goleman (2014) enfatiza que se deve pensar na atenção como um músculo mental, passível de fortalecimento por meio de exercícios como tarefas de memorização e concentração.

Corso et al. (2013), ao realizarem revisão sobre a aprendizagem, afirmam que o aprender relaciona-se às capacidades de planejamento e à regulação da própria atividade em função de determinados objetivos. Ramalho, García-Señorán e González (2011) argumentam sobre a importância do processo de regulação atencional para melhorar a aprendizagem. Em revisão sobre o assunto, os autores mostram que as estratégias de regulação podem ser cognitivas e/ou comportamentais. No primeiro caso, ocorre por meio dos processos de pensamentos, em que os indivíduos podem questionar por possíveis soluções e optarem por aquelas mais adequadas para determinada tarefa. Por sua vez, as estratégias comportamentais buscam favorecer os comportamentos adequados e a inibição dos comportamentos inadequados. 


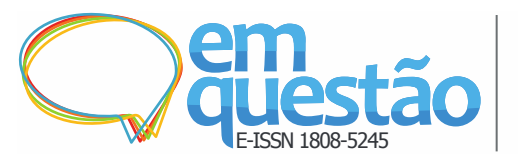

Uma das estratégias cognitivas mais usadas é a de autoinstrução, que permite a redução da impulsividade e o aumento do autocontrole verbal e de conduta motora, por meio da elaboração de metas relacionadas com as atividades propostas e com a forma de atuação em determinadas circunstâncias. Isso possibilita ao indivíduo permanecer atento ao comportamento para avaliar e estabelecer novas metas (RAMALHO, GARCÍA-SEÑORÁN, GONZÁLEZ, 2011).

Dupaul e Stone (2007) argumentam que as bases conceituais, para a intervenção baseada em princípios comportamentais, mostram-se bem documentadas e eficientes no alívio dos problemas de aprendizagem. Elas partem da ideia de combinação de abordagens preventivas quanto mediadoras, que envolvam múltiplos agentes de mediação. A intervenção, apesar de ser recomendada para estudantes com problemas de déficit de atenção, pode ajudar pessoas com dificuldades para prestar atenção, seja pelo barulho do ambiente, seja pelas preocupações oriundas dos interesses da idade, seja pela falta de interesse nos conteúdos entre outras questões. Nesse sentido, supõe-se que os problemas sociais e acadêmicos estão inter-relacionados e vinculam-se à instrução.

A intervenção comportamental deve ter caráter educativo. Sob essa perspectiva, busca-se ensinar as habilidades e os conhecimentos necessários para substituir os comportamentos problemáticos por outros mais aceitáveis. O enfoque educativo da intervenção exige, por sua vez, a implementação de comportamento programático e de planos de apoio ao professor. $\mathrm{O}$ foco da intervenção é aumentar a frequência e a duração dos comportamentos apropriados, em vez de centrar-se na diminuição da disrupção. Nesse sentido, o processo baseia-se no apoio ao comportamento positivo e não em procedimentos reativos e punitivos para mudar o comportamento dos desatentos (DUPAUL; STONER, 2007).

Em relação aos mediadores, DuPaul e Stoner (2007), consideram que as intervenções podem ser realizadas da seguinte maneira:

a) por professores - uso de estratégias de instrução, sistema de reforço por fichas ${ }^{5}$, instrução de habilidades de estudos e contrato de contingências ${ }^{6}$; 


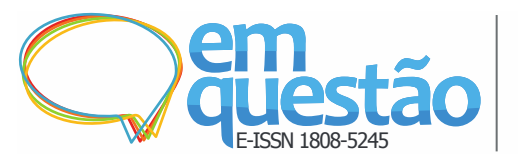

b) por pais - estabelecimento de objetivos, contrato de contingências, reforço no ambiente doméstico, negociação, instrução;

c) por colegas - por instruções sobre a tarefa, treinamento e mediação;

d) por computadores - uso de instrução, exercícios e prática, processamento de texto;

e) autodirigida - por automonitoramento e autoavaliação.

Cabe destacar algumas questões que merecem cuidado durante o planejamento dessas intervenções. São elas: (1) propiciar feedback sobre o comportamento; (2) usar reforços positivos; (3) no caso de solicitação de tarefas independentes, as instruções iniciais devem envolver um número pequeno de tarefas; (4) valorizar produtos e o desempenho acadêmico (finalização de trabalho e nível de exatidão), em vez de comportamentos específicos relacionados à tarefa (atenção à tarefa, permanecer sentado na cadeira); (5) uso de atividades preferidas (escolha de um livro para ler; atividades de desenho), em vez de recompensas tangíveis como adesivos e itens de consumo; (6) monitoramento da intervenção.

\section{Letramento informacional e atenção}

O rápido e grande aumento das fontes de informação em diferentes formatos, a evolução tecnológica e a necessidade de aprendizagem contínua ao longo da vida mostram a relevância do desenvolvimento de competências e habilidades para lidar com a informação - processo conhecido como letramento informacional. Nesse processo, os indivíduos aprendem a identificar um problema, acessar, usar e comunicar uma informação. Como todo processo de aprendizagem, há muitos fatores que o influenciam, em especial as questões psicopedagógicas.

Para o presente estudo, foi realizada uma pesquisa inicial, de natureza exploratória, com o termo "letramento informacional" no portal da Capes e no Google Acadêmico. Esta revisão do tipo narrativa apresenta temática mais 


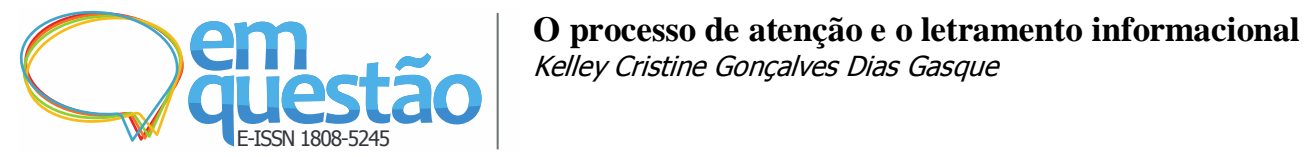

aberta e não requer exaustividade. Em geral, parte de uma questão pouco definida, além de não exigir um protocolo rígido para o desenvolvimento da pesquisa (CORDEIRO et al., 2007). A partir dos resultados apresentados, foram selecionadas pesquisas relacionadas com a área de aprendizagem e o ensino do letramento informacional, com objetivo de mapear, do ponto de vista qualitativo, temas afins.

Foram identificados estudos que propuseram padrões e competências a serem desenvolvidos no ensino superior (MULLINS, 2016); efeitos do LI na aprendizagem (SHAO; PURPUR, 2016; ÇOKLAR; YAMAN; YURDAKUL, 2017; MACEDO, GASQUE, 2018); diagnóstico das competências dos estudantes em LI nos vários níveis de ensinos (FORSTER, 2015; MAYBEE et al. 2015; SAUNDERS et al., 2016); pesquisas sobre questões curriculares (ZINN, GASQUE, 2017; GASQUE, FIALHO, 2017; MOORE et al., 2015); formação de professores e bibliotecários para o LI (DERAKHSHAN; HASSANZADEH; NAZARI, 2015; RIBEIRO; GASQUE, 2015); concepções de ensino-aprendizagem (GASQUE, 2016; GUO; GOH, 2016, CATALANO, 2015); o papel das bibliotecas escolares no LI (MCKEEVER; BATES; REILLY, 2017) e boas práticas para implantação dos programas de LI (WEINER, 2012).

No entanto, apesar do avanço sobre questões vinculadas ao letramento informacional e à aprendizagem, os pesquisadores da ciência da informação ainda precisam compreender melhor os mecanismos de aquisição da informação, bem como as atividades de aplicação do conhecimento. Isso porque, de acordo com Kortelainen, Katvala e Lansman (2017), a sobrecarga de informações do mundo atual traz a necessidade de economizar os recursos mentais, o tempo dos indivíduos e, requer estabelecer prioridades para a busca e a apropriação das informações.

$\mathrm{Na}$ segunda parte do processo de pesquisa, foi realizada a busca de artigos, após login no portal da Capes, no campo de busca simples, com os termos attention and "information literacy”, sem delimitação de data ou de suportes de infomação. Foram apresentados, no display do sistema de busca, 


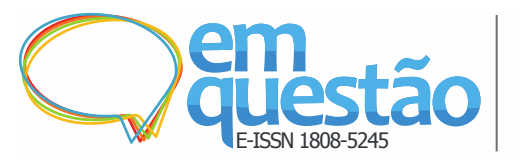

792 resultados, em que 291 eram artigos de periódicos revisados pelos pares e 343 recursos on-line.

A análise dos artigos de periódicos revisados pelos pares mostraram muita repetição de informações e artigos em que o termo attention aparece com sentido de "cuidado", "boa vontade", "gentileza" e "importância", em detrimento do sentido de processo mental. Apenas dois artigos foram considerados pertinentes e relevantes. É importante destacar que os resultados de busca no portal da Capes podem sofrer alterações, de acordo com os contratos de assinatura do governo brasileiro com as instituições, que fornecem as bases de dados. Os resultados mostram que há escassez de pesquisa que associam os dois termos. Uma hipótese provável vincula-se à formação do pesquisador, bibliotecário ou educador, que muitas vezes, não abrange em profundidade os tópicos citados.

A pesquisa de Kortelainen, Katvala e Länsman (2017) buscaram mensurar as características das publicações na internet, que receberam mais atenção dos participantes, levando em conta dois experimentos. O primeiro relacionou-se ao uso de ferramentas de mídia social nas páginas web de revistas científicas e à quantidade de atenção recebida. $\mathrm{O}$ segundo estudo vinculou-se à atenção recebida pelo noticiário de uma rádio, em uma página web. Em ambos os casos, a atenção foi operacionalizada na forma de comentários, curtidas e compartilhamento de postagens na rede social Facebook e, no caso da emissora de rádio, também como comentários publicados no site. Os resultados mostram que nos dois estudos, os ganhos de atenção estavam relacionados à relevância do assunto, ao sentimento de pertença dos participantes na comunidade e ao engajamento nas publicações na web.

A pesquisa de Mcdevitt (2013) questionou como os bibliotecários podem promover o aprendizado dos processos mentais, de ordem superior, vinculados ao letramento informacional e como podem capturar a atenção dos estudantes e envolvê-los no processo de aprendizado. A autora propõe o uso de jogos, de atividades e de tarefas reais baseadas em problemas existentes para solução da questão. 


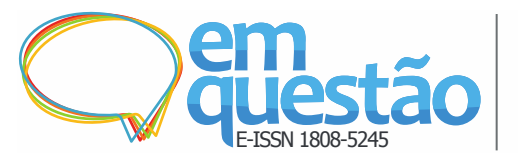

Outros estudos que podem apresentar relevância para a ciência da informação foram encontrados também no portal da Capes com os descritores "attention and internet" e outros descritores como "pesquisa de informação", "uso da informação" juntamente com o descritor "attention", no caso de pesquisas relacionadas ao comportamento informacional humano. A pesquisa de Desjarlais (2013), por exemplo, mostra que a atenção sustentada - capacidade de concentrar a atenção, em uma atividade por um período prolongado de tempo influencia a aprendizagem e a memória, apesar da pouca compreensão dos pesquisadores sobre os fatores comportamentais, que contribuem para a atenção sustentada durante a aprendizagem na internet. No estudo, os estudantes de graduação pesquisaram na internet por informações correspondentes a um tópico de conhecimento de baixo domínio durante 20 minutos, enquanto o olhar deles era registrado. Os vários níveis de atenção eram percebidos por meio da seleção de links dos participantes, do nível de linearidade usado para navegar na internet, pelas estratégias de leitura na web e frequência de implantação de estratégias malsucedidas. Os resultados indicam que o monitoramento atencional durante o uso da internet tem implicações importantes para aprendizagem.

Pesquisa realizada na universidade de Brasília por Vitoriano (2018) mostra que a busca de informações sem relevância, no horário de trabalho, desvia a atenção dos profissionais de tecnologia, causando impactos na produtividade. Sobre isso, a hipótese de Carr (2011) acerca do uso exagerado da internet é que há mudanças na estrutura cognitiva humana. Isso porque as pessoas passam mais tempo na rede, deixando de fazer atividades cognitivas que eram feitas anteriormente sem o uso da internet como leitura de livros e produção de textos, dentre outras. Assim, os circuitos cerebrais que dão suporte a essas antigas funções enfraquecem e começam a se romper. O cérebro recicla os neurônios e as sinapses não usadas para trabalhos mais prementes. $\mathrm{O}$ autor conclui que o indivíduo ganha novas habilidades, mas por sua vez, as antigas são perdidas. Tal questão é bastante expressiva ao considerar que grande parte da busca e do uso da informação ocorrem pela internet. 
De acordo com a Associação de Bibliotecas Americanas (ACRL, 2000), o letramento informacional requer as seguintes competências para determinar a extensão da informação necessária: acessar as informações de forma eficaz e eficiente; avaliar informações e as fontes criticamente; incorporar informações selecionadas na base de conhecimento de uma pessoa; usar as informações de forma eficaz para realizar um objetivo específico; compreender as questões econômicas, jurídicas e sociais que cercam o uso da informação, além de acessar e usar as informações de maneira ética e legal. Resumidamente, de acordo com Mckenzie e Davis (c1986 apud LÁU, 2006), os indivíduos letrados precisam desenvolver a capacidade de:

a) prospecção - compreendida como a capacidade de localizar informações relevantes, filtrá-las e selecioná-las;

b) interpretação - a capacidade de traduzir os dados e informações em conhecimento, insight e compreensão;

c) geração de novas ideias - desenvolver novos insights.

Assume-se, neste artigo, que a criação de novas ideias e a divulgação delas ficariam com maior completude ao usar o termo comunicação da informação. A partir do reconhecimento de que há poucas pesquisas sobre o assunto na ciência da informação, a análise de artigos das áreas de educação e psicologia propiciou sugestões e questões que podem direcionar pesquisas futuras sobre atenção, observando as três competências do letramento informacional.

O objetivo é mostrar algumas possibilidades de pesquisa deste campo para os cientistas da informação, bibliotecários, psicólogos e neurocientistas para fomentar as ações de ensino-aprendizagem do LI, bem como subsidiar o design de bancos de dados.

Quadro 1 - Sugestões de pesquisas a serem realizadas a partir dos padrões de LI

\begin{tabular}{|c|l|}
\hline $\begin{array}{c}\text { Padrões de letramento } \\
\text { informacional }\end{array}$ & \multicolumn{1}{c|}{ Questões } \\
\hline & $\begin{array}{c}\text { Como funciona a atenção na fase de prospecção? } \\
\text { Quais as formas de atenção predominante nessa fase? } \\
\text { Quais as diferenças atencionais dos indivíduos ao manusear as } \\
\text { várias bases de dados? } \\
\text { Quais tipos de fontes de informação sustentam melhor a atenção? } \\
\text { Quais são os principais distratores nessa fase da pesquisa? Como os } \\
\text { indivíduos lidam com eles? }\end{array}$ \\
\hline
\end{tabular}




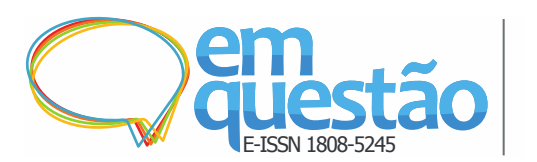

\begin{tabular}{|c|c|}
\hline & $\begin{array}{l}\text { Quanto tempo os indivíduos se engajam na pesquisa de } \\
\text { informação? } \\
\text { Quais são as implicações da pouca atenção no processo de pesquisa } \\
\text { da informação? } \\
\text { Quais tarefas permitem a atenção dividida? }\end{array}$ \\
\hline Uso da informação & $\begin{array}{l}\text { Como os indivíduos detêm a atenção nas diversas tarefas do uso da } \\
\quad \text { informação? } \\
\text { As estratégias de leitura contribuem para ativar a atenção? } \\
\text { Quais as formas de atenção predominante nessa fase? } \\
\text { Como a metaatenção influencia no uso da informação? } \\
\text { Quais são os principais distratores nessa fase da pesquisa? } \\
\text { Como a motivação e a curiosidade influenciam na atenção durante a } \\
\quad \text { fase do uso da informação? } \\
\text { Como ocorre a regulação da atenção nessa fase? } \\
\text { Há diferenças entre especialistas e iniciantes na atenção seletiva? } \\
\text { Os tipos de letras e leiaute influenciam a compreensão do texto? }\end{array}$ \\
\hline $\begin{array}{l}\text { Comunicação da } \\
\text { informação }\end{array}$ & $\begin{array}{l}\text { Qual a diferença de atenção entre especialistas e iniciantes de uma } \\
\text { mesma área? } \\
\text { Quais as estratégias de manutenção da atenção seletiva? } \\
\text { Quais motivos causam a disrupção no processo de pesquisa de } \\
\text { informação? } \\
\text { Quais os procedimentos usados para ativar a atenção nas tarefas de } \\
\text { uso e comunicação da informação? } \\
\text { Como funciona a atenção na produção textual? } \\
\text { Quais são os elementos distratores? } \\
\text { Quais as estratégias para manutenção da atenção? } \\
\text { Como ocorre a leitura e compreensão das normas de documentação } \\
\text { para estruturação do projeto? }\end{array}$ \\
\hline
\end{tabular}
Fonte: Elaborado pela autora.

Portanto, observa-se que as discussões sobre a atenção no letramento informacional ainda são incipientes na ciência da informação. As pesquisas apresentadas mostram que há um longo caminho a percorrer em direção à compreensão dos processos cognitivos, em especial da atenção no letramento informacional. Isso mostra a necessidade de reconhecer a importância do ensinar e aprender melhor no processo de LI.

\section{Conclusões}

A revisão de literatura sobre atenção mostra a necessidade da conscientização dos indivíduos sobre a importância de monitorar a atenção para melhorar a aprendizagem e o desempenho, de forma geral. A atenção sustentada potencializa o processamento mental, fortalece as sinapses e expande as redes neurais nas tarefas realizadas. 
O letramento informacional refere-se ao processo de aprendizagem necessário para o desenvolvimento de competências informacionais. Tais competências relacionam-se à aquisição dos conteúdos de LI que envolvem a busca, o uso e a comunicação da informação de maneira eficaz e eficientemente. A complexidade desse processo exige dos aprendizes a atenção seletiva e a manutenção da atenção sustentada para desempenhar as tarefas. No entanto, há grande escassez de pesquisas sobre esse assunto.

As teorias sobre atenção ainda suscitam debates sobre quais delas explicam melhor a atenção seletiva. As teorias mais recentes sustentam-se na ideia de partilha de recursos limitados da atenção e tentam explicar como várias tarefas são realizadas ao mesmo tempo. A literatura mostra que a atenção pode ser regulada e aperfeiçoada ao longo do processo de aprendizagem a partir do ensino de estratégias atencionais e metaatencionais do tipo cognitivas ou comportamentais. Essas estratégias, de natureza autorreguláveis possibilitam que as pessoas monitorem e possam melhorar o foco da atenção.

A análise de artigos sobre a atenção das áreas de educação e psicologia propiciou a transferência de sugestões e levantamento de questões que podem ser de interesse da área de ciência da informação, considerando as principais fases do letramento da informação, quais sejam, prospecção, uso e comunicação da informação.

Os temas sugeridos são, em grande maioria, de caráter generalista, mas com possibilidade de subsidiar o processo de LI e possibilitar intervenções mais assertivas durante realização de tarefas informacionais. Por fim, reconhece-se a importância de compreender e aprimorar o ensino e as estratégias de aprendizagem para o letramento informacional.

\section{Referências}

ASSOCIATION OF COLLEGE AND RESEARCH LIBRARIES. Information literacy competency standards for higher education. Chicago: ALA, 2000. CARR, Nicholas. A geração superficial: o que a internet está fazendo com os nossos cérebros. Rio de Janeiro: Agir, 2011. 
CATALANO, Amy. The effect of a situated learning environment in a distance education information literacy course. Journal of Academic Librarianship, New York, v. 41, n. 5, p. 653-659, 2015.

CORDEIRO, Alexander Magno et al. Revisão sistemática: uma revisão narrativa. Revista do Colégio Brasileiro de Cirurgiões, Rio de Janeiro, v. 34, n. 6, p. 428-431, nov./dez. 2007.

CORSO, Helena Vellinho et al. Metacognição e funções executivas: relações entre os conceitos e implicações para a aprendizagem. Psicologia: teoria e pesquisa, Brasília, v. 29, n. 1, p. 21-29, 2013.

ÇOKLAR, Ahmet Naci; YAMAN, Nihal Dulkadir; YURDAKUL, Işıl Kabakç1. Information literacy and digital nativity as determinants of online information search strategies. Computers in Human Behavior, Oxford, v. 70, p. 1-9, 2017.

DE-NARDIM, Maria Helena; SORDI, Regina. Um estudo sobre as formas de atenção na sala de aula e suas implicações para a aprendizagem. Psicologia \& Sociedade, Belo Horizonte, v. 19, n. 1, p. 99-106, jan/abr. 2007.

DERAKHSHAN, M.; HASSANZADEH, M.; NAZARI, M. Developing information literate librarians: a study of LIS academics pedagogical approaches in the development of information literacy competencies. Journal of Academic Librarianship, New York, v. 41, n. 6, p. 777-785, 2015.

DESJARLAIS, Malinda. Exploration behaviours and recovery from unsuccessful actions differ between learners with high and low levels of attention. Computers in Human Behavior, Oxford, v. 29, n. 3, p. 694-705, 2013.

DUPAL, George; STONER, Gary. TDAH nas escolas. São Paulo: M. BOOKS, 2007.

EYSENCK, Michael W.; KEANE, Mark T. Manual de psicologia cognitiva. 5. ed. Porto Alegre: Artmed, 2007.

FORSTER, Marc. Six ways of experiencing information literacy in nursing: The findings of a phenomenographic study. Nurse Education Today, [s.l.], v. 35, n. 1, p. 195-200, 2015.

GASQUE, Kelley Cristine Gonçalves Dias. Comportamento, letramento informacional e pesquisas sobre o cérebro: aplicações na aprendizagem. Informação em Pauta, Fortaleza, v. 2, out. 2017. Número especial.

GASQUE, Kelley Cristine Gonçalves Dias; FIALHO, Janaina. Letramento informacional e Currículo. Ponto de Acesso, Salvador, v. 11, n. 2, p. 70-89, ago. 2017.

GASQUE, Kelley Cristine G. D. Information literacy for inquiry-based learning. 
Transinformação, Campinas, v. 28, n. 3, p. 253-262, 2016.

GOLEMAN, Daniel. Foco: a atenção e o seu papel fundamental para o sucesso. Rio de Janeiro: Objetiva, 2014.

GUO, Yan Ru; GOH, Dion Hoe-Lian. Evaluation of affective embodied agents in an information literacy game. Computers \& Education, Oxford, v. 103, p. 59-75, 2016.

HERBERT, Simon. Designing organizations for an information-rich world. Baltmore: The Johns Hopkins Press, 1971.

KORTELAINEN, Terttu; KATVALA, Mari; LÄNSMAN, Anni-Siiri. Attention and altmetrics. Information Research, Borás, v. 22, n. 1, p. 1-9, 2017.

JAMES, Willian. The principles of psychological. New York: Henry Holt and Company, 1890. v. 2.

LAU, Jesus. Guidelines on Information literacy for Lifelong learning.. Edinburgh: IFLA, 2006.

LIMA, Ricardo Franco de. Compreendendo os mecanismos atencionais. Ciências e Cognição, Rio de Janeiro, v. 6, n. 5, p. 113-122, 2005.

LIMA, Ricardo Franco de; TRAVAINI, Paula Pinheiro; CIASCA, Sylvia Maria. Amostra de desempenho de estudantes do ensino fundamental em testes de atenção e funções executivas. Revista Psicopedagogia, São Paulo, v. 26, n. 80, p. 188-199, 2009.

MACEDO, M. M.; GASQUE, Kelley Cristine G. D. A influência do Letramento Informacional na aprendizagem de estudantes na educação básica. Revista Ibero-americana de Ciência da Informação, Brasília, v. 11, n. 1, p. 5-22, 2018.

MAYBEE, Clarence et al. "It's in the syllabus": identifying information literacy and data information literacy opportunities using a grounded theory approach. Journal of Academic Librarianship, New York, v. 41, n. 4, p. 369-376, 2015.

MCDEVITT, Theresa. Everyone likes a challenge: getting students attention with interactive games e authentic assignments. Pennsylvania Libraries: Research and Practice, Indiana, v. 1, n. 2, p. 149-161, 2013.

MCKEEVER, Christine; BATES, Jessica; REILLY, Jacqueline. School library staff perspectives on teacher information literacy and collaboration. Journal of Information Literacy, Loughborough, v. 11, n. 2, p. 51-68, 2017.

MOORE, Carrie et al. Integrating information literacy, the POGIL method, and ipads into a foundational studies program. Journal of Academic Librarianship, New York, v. 41, n. 2, p. 155-169, 2015. 
MULLINS, Kimberly. IDEA Model from Theory to Practice: integrating information literacy in academic courses. Journal of Academic Librarianship, New York, v. 42, n. 1, p. 55-64, 2016.

RAMALHO, Joaquim; Garcia-Senoran, M.; GONZALEZ, Salvador. Autoinstruções: estratégias de regulação atencional de THDA. Psicologia: reflexão e crítica, Porto Alegre, v. 24, n. 1, p. 180-185, 2011.

RATEY, John J. O cérebro: um guia para o usuário. Rio de Janeiro: Objetiva, 2002.

RIBEIRO, Leila Alves Medeiros; GASQUE, Kelley Cristine Gonçalves Dias. Letramento Informacional e Midiático para professores do século XXI. Em Questão, Porto Alegre, v. 21, n. 2, p. 203-221, 2015.

SAUNDERS, Laura et al. Assessing graduate level information literacy instruction with critical incident questionnaires. Journal of Academic Librarianship, New York, v. 42, n. 6, p. 655-663, 2016.

SHAO, Xiaorong; PURPUR, Geraldine. Effects of Information Literacy Skills on Student Writing and Course Performance. Journal of Academic

Librarianship, New York, v. 42, n. 6, p. 670-67, 2016.

STERNBERG, Robert. Psicologia cognitiva. Porto Alegre: Artmed, 2000.

VITORIANO, Maria Albeti Vieira. Impactos da busca de informações não relevantes na produtividade de profissionais de tecnologia da informação. 2018. Tese (Doutorado em Ciência da Informação) - Faculdade de Ciência da Informação, Universidade de Brasília, Brasília, 2018.

WEINER, Sharon A. Institutionalizing Information Literacy. Journal of Academic Librarianship, New York, v. 38, n. 5, p. 287-293, 2012.

ZINN, Alexandra César; GASQUE, Kelley Cristine Gonçalves Dias. A construção de um programa de letramento informacional e arte educação.

Revista Digital de Biblioteconomia e Ciência da Informação, Campinas, v. 15, n. 1, p. 171-188, 2017. 


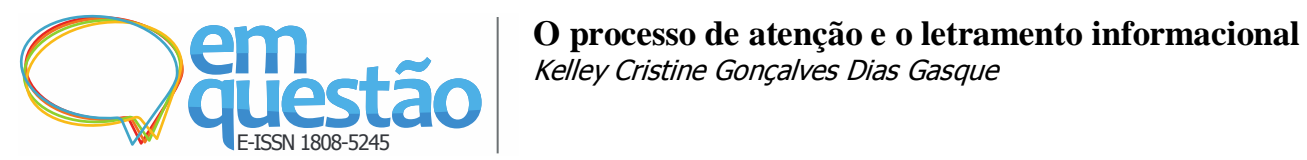

\title{
Attention process and information literacy
}

\begin{abstract}
This article is a literature review of an exploratory and selective nature, which aims to map studies related to information literacy and attention process, to raise possible research topics, and to identify ways to regulate attention. Part of the assumption is that attention is a fundamental process in the learning of information literacy contents, as well as in the development of activities and research tasks. The results show that the maintenance of attention is fundamental to human's cognitive tasks, and consequently, to Information Literacy. Research in the field of information science is incipient and requires investment. In general, it is possible to improve focus through cognitive or behavioral regulation.
\end{abstract}

Keywords: Information literacy. Attention. Self-regulation. Learning.

Recebido: 07/11/2018

Aceito: 20/02/2019

${ }^{1}$ Stroop Color Word Test avalia a atenção seletiva visual e a inibição cognitiva, isto é, a capacidade de inibir respostas automáticas

${ }^{2}$ O Trail Making Test empregado para avaliar a atenção (Parte A) e a flexibilidade mental e a capacidade de alternar entre categorias cognitivas (Parte B)

3 Testes de Cancelamento (TC) são instrumentos que avaliam a atenção sustentada na modalidade visual.

${ }^{4}$ A Tower of London (TOL) avalia a capacidade de planejamento e resolução de problema.

5 Utilizam-se fichas como reforço secundário para recompensa, por exemplo, estrelinhas, adesivos ou pontos.

${ }^{6}$ Técnica de manejo comportamental que envolve a negociação entre professor e aluno.

7 MCKENZIE, Jamie; DAVIS, Hilarie Bryce. Filling the tool box: classroom strategies to engender student questioning. The Educational Technology Journal, Englewood Cliffs, 1986. Apud Láu (2016). 